\title{
Technology led to more abstract causal reasoning
}

\author{
Peter Gärdenfors ${ }^{1,2,3}$ - Marlize Lombard ${ }^{2,3}$ (D)
}

Received: 14 November 2019 / Accepted: 25 June 2020 / Published online: 6 July 2020

(c) The Author(s) 2020

\begin{abstract}
Many animal species use tools, but human technical engagement is more complex. We argue that there is coevolution between technical engagement (the manufacturing and use of tools) and advanced forms of causal cognition in the human (Homo) lineage. As an analytic tool, we present a classification of different forms of causal thinking. Human causal thinking has become detached from space and time, so that instead of just reacting to perceptual input, our minds can simulate actions and forces and their causal consequences. Our main thesis is that, unlike the situation for other primate species, an increasing emphasis on technical engagement made some hominins capable of reasoning about the forces involved in causal processes. This thesis is supported in three ways: (1) We compare the casual thinking about forces of hominins with that of other primates. (2) We analyze the causal thinking required for Stone Age hunting technologies such as throwing spears, bow hunting and the use of poisoned arrows, arguing that they may serve as examples of the expansion of casual cognition about forces. (3) We present neurophysiological results that indicate the facilitation of advanced causal thinking.
\end{abstract}

Keywords Causal cognition · Stone tool technology $\cdot$ Hominins $\cdot$ Forces $\cdot$ Cognitive evolution $\cdot$ Stone napping $\cdot$ Cognitive archaeology

\section{Humans have an exceptional relationship with technology}

In this article, we argue that there is coevolution between the manufacturing and use of tools and advanced forms of causal thinking. Human causal thinking has become increasingly abstract in the sense that it is detached from space and

Peter Gärdenfors

Peter.Gardenfors@lucs.lu.se

1 Cognitive Science, Department of Philosophy, Lund University, 22100 Lund, Sweden

2 Palaeo-Research Institute, University of Johannesburg, P.O. Box 524, Auckland Park 2006, South Africa

3 Stellenbosch Institute for Advanced Study (STIAS), Wallenberg Research Centre at Stellenbosch University, Stellenbosch 7600, South Africa 
time, so that instead of just reacting to perceptual input, our minds can simulate, amongst other things, the forces involved in actions and other events and the causal consequences of such forces. Our main thesis is that, unlike the situation for other primate species, tool production and use were one of the factors that stimulated the ability for these forms of reasoning in later hominins.

Human relationships with technology are stronger than in any other species, and the manufacture and use of tools have become a defining characteristic of our species (Ambrose 2001; Barham 2013; Boivin 2008; Lombard 2016; Shea 2017). We extend our bodies, and by implication our minds, through the 'prosthetic' use of technology (Malafouris 2013), and by the understanding and application of the underlying abstract physical, chemical and biological principles of our technologies (Gärdenfors and Lombard 2018). Consequently, tracing the development of our cognitive and bodily relationships with different forms of technology is central to developing an understanding of how we evolved the capacity for abstract causal thinking.

Many animal species also use tools, but human technical engagement is more complex. Shumaker et al. (2011: 223-225) identify four ways in which human tool making and use are more or less unique.

1. Non-human animals do not typically use tools to make tools.

2. Non-humans other than great apes rarely use tools in combinations for a single task.

3. Non-humans rarely cooperate in using or making tools.

4. Non-humans power their tools only with gravity and their own energy.

When discussing causal cognition in terms of tool use and how our minds represent the world, it is useful to distinguish between cued (externally signaled) and detached mental representations (Gärdenfors 2003). A cued representation refers to something, or a property of something in the current or recently experienced external situation of the experiencer. When, for example, a chimpanzee uses two stones to crack nuts, it represents one of them as support (anvil) and the other as the hammerstone. By contrast, detached representations stand for objects or events that are not present in the subject's current or recent external context and so could not directly trigger the representation. For example, when a chimpanzee plans to fish for termites with a stick, it walks away and breaks off a twig from a nearby tree and prepares it to fish with (Sanz et al. 2004). Forming a mental image of the prepared stick is a detached representation that becomes part of the plan of the chimpanzee.

Being able to use a detached representation requires that one can suppress the sensations one has for the moment; otherwise they will come into conflict with the representation. Glenberg (1997) argues that detached representations put reality in quarantine. An individual, human or non-human, that has detached representations can represent the world in an inner space where consequences of different actions or events can be simulated (Grush 1997; Gärdenfors 2003; Decety and Grèzes 2006). Such simulations form what we normally call imagination 
(Gärdenfors 2003). Spreng and Grady (2010: 1112), argue that "remembering one's past, imagining one's future, and imagining the thoughts and feelings of others ... are similar in that they all involve simulating an experience that is distinct from stimulus-driven behaviour". We aim to show that such simulations are also central for abstract causal reasoning, and that its development in the later phases in the Homo lineage may have been stimulated, amongst other things, by technologies operating across space and through time.

To analyze the interplay between tool making and use on the one hand, and causal thinking on the other, we first present a summary of the seven grades of causal cognition from Lombard and Gärdenfors (2017). We focus in this article on the evolution of grade 6-understanding abstract causes. In particular, our aim is to show that this grade involves understanding the role of non-animate forces.

\section{A classification of causal cognition}

Table 1 below is an extension of earlier classifications by Gärdenfors (2003) and Woodward (2011). The classification was developed for a study of the evolution of tracking behavior, but here we apply it to the causal reasoning involved in technical engagement. (For further discussion and examples of the seven grades see Lombard and Gärdenfors (2017) and Gärdenfors and Lombard (2018)).

It is, of course, rather hopeless to try to date when each of the seven grades we propose emerged during hominin evolution. Our arguments are instead based on comparisons between the cognitive capacities that are required for the different forms of causal cognition. We rely on the following methodological principle:

Cognitive parsimony: If the cognitive capacities required for an activity or technique $\mathrm{A}$ is a subset of those required for an activity or technique $\mathrm{B}$, then $\mathrm{A}$ is evolutionarily prior to $\mathrm{B}$.

Even though this principle does not say anything about dating, it makes it possible to argue that one type of activity is evolutionarily older than another.

This principle entails that the grades of causal reasoning do not necessarily follow a unilinear evolutionary trajectory. Aspects of each type of thinking may have evolved parallel to one another, or are still evolving within continuing co-evolutionary feedback loops. Within each grade of causal understanding there might be several levels of complexity that developed at different times in different places and/ or in different hominin populations (Lombard and Gärdenfors 2017). For example, basic, conspecific mindreading skills (Grade 3 below) might have been acquired early on in our evolution. However, enhanced levels of human mindreading, or theory of mind, that enable us to cope with current complex societies, might only have evolved at a later stage, i.e., after we were able to understand and interpret the behaviours of non-conspecifics or Grade 5 causal understanding (Tomasello et al. 2005; Gärdenfors 2007). Thus, a newly identified grade of causal understanding does not automatically imply that all or some aspects of the previously identified grade stopped its evolutionary trajectory.

The seven grades should be seen as a development involving several types of cognitive representations. One aspect is the distinctions between understanding 
Table 1 Seven grades of causal reasoning (adapted from Lombard and Gärdenfors 2017)

1. Individual causal understanding. The first grade involves a direct connection between a bodily action that an individual executes and the resulting effect. A typical example is a baby kicking its foot, learning the connection between motor commands and the resulting actions. In this case both the cause and the effect are directly perceived. The result is that the individual experiences its own agency

2. Cued dyadic-causal understanding. This grade involves two individuals who take turns in performing a similar action. One example is two children on a seesaw. The motor forces behind the other individual's actions are not directly perceived, but they are inferred via a mapping onto the forces involved in one's own actions. Neurologically, this is accomplished with the aid of mirror neurons (Rizzolatti and Craighero 2004). Thus, I understand that the action of the other causes an effect because it gives the same result as my own action. On this grade, one individual also understands the agency of the other (Leslie 1995)

3. Conspecific mindreading. Humans understand how our desires, intentions and beliefs lead to different kinds of actions (Gärdenfors 2003, 2007). One example of this grade is gaze following, i.e., understanding that if someone is looking firmly in a particular direction, there is something worthy of attention in that direction. In other words, the onlooker infers that there is a cause for the gaze direction, even if the onlooker itself cannot perceive the cause. On this grade, attention, desires, intentions and beliefs are seen as 'mental forces' causing the action of the other (Gärdenfors 2003)

4. Detached dyadic-causal understanding. Sometimes we do not perceive the actions of somebody else, but only the traces of them. An example is the tracks of a person or animal in the sand by the river. The cause is detached in time from the present situation. I don't see the person or animal, but conclude that somebody's agency in the past is the cause of the tracks (see also Martin 2014). This is a cognitive extension of grade 2

5. Causal understanding and mindreading of non-conspecifics. We sometimes have a dyadic-causal understanding of the actions and intentions of other species, although their motor actions and cognitive processes are different from ours. For example, when I see an animal track, I can infer the cause, since I recognize the track as that of a gemsbok (grade 4). With increased experience, I may also be able to infer the mental states of the animal, for example, if I see blood in the tracks and that the gemsbok is limping, I can, first, draw the conclusion that the gemsbok is hurt by using detached dyadic-causal reasoning, and, second that it is in pain by using a form of empathy mirroring my own experiences of being hurt. This grade relies on a combination of the cognitive capacities of grades 3 and 4 , but does not imply that the evolution of those types of reasoning came to an end

6. Inanimate causal understanding. Mind-reading of conspecific and non-conspecific individuals involve causes that are parts of what is attributed as the mental state of an individual. The most abstract causes are forces-physical, chemical and biological - that are generated by inanimate objects. For example, I see a twig being stuck in the resin on the trunk of a tree or I see an animal being stuck in the mud of the drying waterhole. Again, I don't perceive the cause, but I infer the forces from the resin or the mud, using memories of similar cases of adhesion. Unlike the previous cases, there is no animate agent that performs an action

7. Causal network understanding. We suggest that the most complex grade of causal cognition is the understanding of how domain-specific causal node sets connect or link to inter-domain causal networks (Tenenbaum and Niyogi, 2003). Such networks have been modelled by Bayesian methods (Gopnik et al. 2004), and the most advanced form of this kind of reasoning is science (Gopnik et al. 1999). Thus, with causal network understanding I am able to abstract the knowledge gained from one domain and apply it to another by imagining how past scenarios can be used in the future to solve a range of unrelated problems innovatively

the consequences of one's own actions, the actions of others, and the 'actions' of non-living objects. Another distinction is between perceivable causes and causes that must be inferred. A third set of distinctions is the one between physical actions of others, components of the minds of others (mind-reading) functioning as causes, and physical forces involved in the 'actions' of non-living objects. The 
seven grades that we identify are derived by combining these distinctions in different ways.

Applying the principle of cognitive parsimony, the difference between grades 3 and 4 on the one hand, and grade 5 on the other, is gradual and depends to a large extent on the experience of the behavior of other species. Grade 4 depends on the capacity to entertain two mental representations at the same time, that is, the current perceptual state of seeing the tracks together with my detached simulation of the person or animal being present in the field at another point in time (Leslie 1987).

There is evidence that non-human animals manage causal cognition on grades 1-3 (Woodward 2011; Shumaker et al. 2011; Lombard and Gärdenfors 2017). Several experiments and observations indicate that monkeys and apes do not reach grade 4, since they do not infer physical causes from their effects (Cheney and Seyfarth 1990; Povinelli 2000, but see Mulcahy and Call 2006). Other experiments have, however, challenged the claim that reasoning from effects to non-present causes is unique to humans. For example, a study by Völter and Call (2014) found that apes can make use of a trail left by a leaking yoghurt cup that was placed out of their sight, to locate the cup. On the other hand, they did not use the trail when it did not match the type of food that is displaced. Our position is supported by an experiment by Civelek et al. (2020) that examined inferences about unseen causes in children and chimpanzees. The subjects saw a reward being dropped through an opaque forked-tube into one of two cups. An auditory cue signaled which of the cups contained the reward. In one condition, the cue followed the dropping event, indicating that the sound was caused by the reward falling into the cup, and in another condition, the sound preceded the dropping event. Four-year-old children performed better in the causal condition than the other one, suggesting that they engaged in casual reasoning on grade 4. In contrast, chimpanzees and three-year-olds performed at chance in both conditions, suggesting that they did not reason about the unseen cause.

The available empirical evidence suggests that apes do not reach grades 5-7 (Gärdenfors and Lombard 2018; Lombard and Gärdenfors 2017). It seems that nonhuman animals understand causation only in terms of agency, while humans today can also reason about causes in a detached way via forces that operate across space (action at a distance) and time. When the seven-grade model for causal reasoning was used as part of a multi-modal comparative exploration in capuchin rock pounding, chimpanzee nut cracking and Lomekwi 3 knapping, only basic levels of grade 3 were reached (see Lombard et al. 2019). Here, however, we focus on the later grades of causal cognition and their relevance to recent human cognitive evolution.

Some experiments suggest that corvids may be more advanced causal reasoners than primates (e.g. Taylor et al. 2009). In an experiment with New Caledonian crows, Jelbert et al. (2019) showed that the birds could infer which of two objects that were suspended from strings was the heavier by observing the movements of the objects when a fan was turned on and moved the light object more than the heavy one. The birds picked the heavier object in $73 \%$ of the cases. This is an example of a tendency to infer a causal factor-the weight - that leads to an effect- the movement in the breeze, which is an example of grade 4 causal reasoning. A speculative hypothesis, which is in line with our arguments concerning hominins, is that the 
New Caledonian crows' extensive tool use have led them to develop comparatively advanced causal reasoning.

In Lombard and Gärdenfors (2017), we studied the evolution of grade 4, and to some extent grade 7, in relation to the cognitive capacities involved in tracking and the archaeological evidence for these. And in forthcoming research we will focus on the co-evolution of causal cognition and theory of mind (mainly involved in grades 3 and 5). In this article we instead focus on the evolution of grade 6. Our main thesis, which is developed in the following sections, is that causation is understood in terms of force dynamics (Gärdenfors and Warglien 2012; Leslie 1995; Povinelli 2000; Wolff 2007; Wolff and Shepard 2013; Wolff and Thorstad 2017), which is an extension of the bodily agency of the individual that leads to new forms of causal representations that are detached in space and time. Furthermore, we argue that the evolutionary mechanism behind this extension is driven by our technological engagement in tool making and tool use.

\section{Understanding physical forces}

\section{Human reasoning about physical forces}

The human capacity to understand the role of physical forces, not just the forces involved in your own or other individuals' actions, develops early in infants. Michotte (1963) showed that if one object moving on a screen collided with another object and the other object started moving in the same direction, then adults perceived the launching of the second object as caused by the movement of the first. In contrast, if the second object only started moving half a second after the collision, the delay destroyed the impression of causality. Leslie and Keeble (1987) performed Michotte's experiments with six-month-old infants, showing that they reacted differently to the two event types. Leslie (1995) concludes that infants have a special system in their brains for mapping the 'forces' of objects. Further psychological evidence (Wolff 2007, 2008; Wolff and Shepard 2013; Wolff and Thorstad 2017) supports that people can directly perceive the forces that control different kinds of motion. In other words, the sensory input generated by the movements of an individual (or an object) is sufficient for the brain to calculate the forces that lead to the movements. The process is automatic: people cannot help but seeing the forces.

Wolff (2007) showed that adults can combine physical causes in their reasoning. For example, they can estimate the combined forces of a boat motor and the wind and their effects on how the boat crosses a lake. Göksun et al. (2013) extended this to a study of 3-5-year-olds who, in addition to one-force events, were asked to predict the path of a ball that was influenced by two forces that were combined to reflect force dynamics patterns of 'cause', 'enable' and 'prevent'. They showed that while children were successful in their causal reasoning about the one-force events, they were selectively attentive to a second force, incorporating it only if both forces move in the same direction. The older the children, the more successful their reasoning about the effects of the second force (George et al. 2019). These experiments indicate that human abstraction and reasoning about physical forces develop with 
experience over age, although the general system for perceiving forces as causes is present already at an early age.

\section{Non-human primate reasoning about physical forces}

It seems that non-human primate reasoning about forces is less developed compared to that of humans. For example, in his early experiments on chimpanzee planning, Köhler (1917) observed that apes had great difficulties in stacking boxes. He notes that when Sultan, the best problem solver among the chimpanzees, tried to put a second box on top of a first, instead of doing so "as might seem obvious, began to gesticulate with it, ... he put it beside the first, then in the air diagonally above, and so forth". After similar observations on other apes, Köhler (1917:149) concludes that "there is practically no statics to be noted in the chimpanzee" (also see Tomonaga et al. 2007; Cacchione et al. 2009).

Povinelli (2000) performed a series of experiments also indicating that chimpanzees and other primates are limited in their reasoning about the effects of gravitation on objects. These experiments have been followed by others (Call 2010; Hanus and Call 2008; Martin-Ordas et al. 2008; Penn and Povinelli 2007), generating an extended debate (see Seed et al. 2009, 2011). Povinelli and Penn (2011:77) conclude that "only humans are capable of second-order relational reasoning, and only humans, therefore, have the cognitive machinery that can support higher-order, theory-like, causal relations". Johnson-Frey (2003:201) also writes: "Comparative studies of chimpanzee tool use indicate that critical differences are likely to be found in mechanisms involved in causal reasoning rather than those implementing sensorimotor transformations".

A potential counterexample comes from the recent work of Matsuno and Tomonagab (2017), who show that chimpanzees are subject to the same visual illusion, called causal capture, as humans. The illusion is caused by a bistable visual motion of objects that bounce against each other. This result shows that the perceptual mechanism behind the interpretation of the bouncing is evolutionarily shared between humans and chimpanzees. However, such perceptual mechanism need not involve any detached understanding of forces. Also in Boesch et al.'s (2017) comparative study on nut cracking in humans and chimpanzees, they found that humans understood how to apply force using hammerstones to extract numerous nut species. Yet, the chimpanzees in their study group only ever applied such force to Panda nuts, although they regularly eat kernels from hard Irvingia nuts using their teethsometimes causing tooth breakage because of the force required. Chimpanzees in other groups and regions cracked different nut types with hammerstones (Hannah and McGrew 1987; Morgan and Abwe 2006; Whitesides 1985), but to our knowledge the same group of chimpanzees never or seldom uses hammerstones to extract a range of food sources. This is a good example of how humans, compared to chimpanzees, have a more abstract causal understanding of tool-assisted force application, allowing us to apply similar solutions to a wider range of subsistence problems. We submit that by adding the ability to mentally represent detached forces-and not just actions - as causes, the human mind evolved to extend its capacities to reason 
and to plan beyond that of other primates; a development that was driven (at least in part) by technical engagement.

\section{Technology made us causal reasoners about force}

A key evolutionary question then becomes why only humans evolved causal thinking that is based on forces? The answer, we suggest, derives from the fact that the Homo lineage is the only group who can be defined based on their intimate relationship with technology (Shea 2017).

\section{Early hominin tool use}

An early extension of causal cognition probably appeared when hominins started to produce stone flakes to cut meat from scavenged carcasses. Currently, the oldest evidence for such behavior is the Lomekwi 3 tools from Kenya at about 3.3 million years ago (Harmand et al. 2015), before the earliest known Homo fossils of about 2.8 million years old (Villmoare et al. 2015).

Lombard et al. (2019) found that grades 1 and 2 causal understanding are necessary, to knap the bipolar flakes from Lomekwi 3, and planning is required to transport the flakes when used elsewhere, as argued for the Oldowan (Gärdenfors and Osvath 2010). Yet, the understanding of forces involved in knapping could still be immediate and not detached in such early stone knapping and flake use. On the other hand, procuring material for the knapping process typically involves detached representations.

\section{Tools as amplifiers}

Tools extend your agency, and making it possible to act at a distance and to alter the force patterns generated by your body. A classic example is the stick of a blind person (Merleau-Ponty 1962). Although she can feel her surroundings with her hands, with the aid of the stick she extends her peripersonal space-the space within which she can act directly (Maravita and Iriki 2004). The stick allows her to detect obstacles that are beyond the reach of her hands. It is a double prosthetic; the force patterns of the stick also serve to generate sounds, and its echo informs on the layout of the environment.

Tools function as amplifiers. First, many tools amplify actions. A hammer-stone amplifies the pounding power of hands. A stick used to fish for termites or honey extends the poking of fingers. Using a stone flake to cut meat amplifies the cutting power of teeth. Secondly, as the blind person's stick illustrates, tools can amplify perception. Modern tools such as eyeglasses, binoculars, microscopes, hearing aids, etc., are also sensory amplifiers. Thirdly, some tools amplify cognition. Abstractions in the form of words, numbers and other symbolic systems amplify memory, planning and communication. An example of this is using tallying strokes or marks to count days, thereby enhancing memory and planning capacities (also see Iriki 
and Taoka 2012: 13 Fig. 1c in for corresponding mechanics, detector and thought levels.)

\section{Extending action fields and action control}

We next turn to how tools extend our potential to act across space. The visual field of primates is divided into peripersonal and extrapersonal space. The peripersonal space (the region within reach around the body), allows an individual to see its field of action. Tool use extends the peripersonal space, in humans and primates (Cléry et al. 2015; Maravita and Iriki 2004). By having the reactive fields of hand neurons extended to the hand together with the tool, tools are assimilated into the body schema (Maravita and Iriki 2004). Tool use thereby causes part of the extrapersonal space to be remapped as 'within reach'. Neurologically, this drives interactions between the intraparietal sulcus and the temporoparietal junction, as a result there is a blurring between the neural representations of the hand and the tool (Iriki 2006).

Arbib et al. (2009) claim that the neural consequence of tool use is not restricted to changes in the representation of peripersonal space-it also involves a mapping that captures how movements of the hands are transformed into actions of the tool's end-effector (the part of the tool used to act upon another object). The tool typically changes the form of the actions we perform. Consider tightening a nut and bolt with either the hand or a pair of pliers. The jaws of the pliers function just like the opposed fingers of the hand, but the hand movements we perform in the two cases are quite different. Arbib et al. (2009, p. 443) write that this "suggests that some abstract effector-independent representation provides criteria for success in achieving a goal that guides learning". They also put forward "the hypothesis that, as we turn from grasping an object to using a tool to grasp an object, many neurons will code the movement of the end-effector of the tool, rather than that of the hand, in a reference frame centered on the relevant affordance of the manipulandum" (ibid, p. 444).

Umiltà et al. (2008) performed an experiment, involving normal and reverse pliers that illustrates this hypothesis: With normal pliers the hand is first opened and then closed to grasp an object, while with reverse pliers the hand is first closed and then opened. Grasping an object with the two tools required opposite sequences of hand movements to achieve the same grasping goal. Umiltà et al. (2008) then checked whether the firing of a neuron correlated better with hand motion or end-effector motion. They found that neurons that discharged when the hands was opening with normal pliers discharged when the hand was closing reverse pliers were used, supporting the hypothesis that the neuron react to the action of the end-effectors and not to the hand actions.

A synthesis of neuro-scientific work on the cognitive processes that give rise to human causal reasoning revealed that prefrontal areas show domain independence during high-level causal reasoning, while areas within the temporal, parietal, and occipital lobes exhibit evidence of domain dependence in such reasoning (e.g. Krawczyk 2012). Neuropsychological and neuroimaging studies further indicated that human behaviors associated with complex tool use is facilitated by functionally 
specialized networks involving temporal, parietal and frontal areas (Johnson-Frey 2004). Goldenberg and Spatt (2009) found that parietal lesions impaired mechanical problem solving, i.e., understanding how one tool interacts with other tools, which is essential for conceptualizing and using complex technologies.

In sum, a shared and integrated frontoparietal network is key in human spatial attention, space-related behaviors, fluid intelligence and for high-level causal reasoning related to the use of complex technology (e.g., Barbey et al. 2012; Doricchi et al. 2008; Hampshire et al. 2010). Such a network forms the basic neural mechanism for mental simulations through space-time, and seems to be uniquely human (Bruner 2010).

\section{Seeing oneself from the outside}

Including tools in peripersonal space may be a first step towards 'self-objectification' (Iriki 2006). The subject can then shift perspective and perceive its body as having functions similar to that of tools. In this way, the agent splits its awareness into a 'subjective self' and an 'objective self-body' (compare Fig. 2 in Iriki 2006: 663). An agent can thereby attend to its own actions from an outside, detached perspective at the same time as directly experiencing the actions of its body. Once this perspective is in place, it is possible for an agent to perceive the force patterns of their own body.

Iriki (2006) suggests that humans have a genuinely voluntary intention system that can perform, or even just imagine, actions divorced from immediate context. For example, there is an increasing decoupling of problem and solution in modular tool use (e.g. Haidle et al. 2015). This system not only enables us to mirror and understand the intentions of other people, it endows us with a fully developed theory of mind. The medial prefrontal cortex, temporoparietal junction and temporal pole, together with a few additional structures, might carry out this function (Iriki 2006). In support of this, Spreng and Grady (2010) found that autobiographical memory, prospection, and theory of mind shared a common pattern of brain activity, including midline structures in the frontal and parietal lobes associated with the default mode network - a highly interconnected core set of brain structures including the midline frontal and parietal structures, medial and lateral temporal lobes, and lateral parietal cortex. Such coordination is sufficient for producing self-relevant stimulusindependent thought, and is consistent with brain activity related to self- and otherreferential thought (D'Argembeau et al. 2008; Johnson et al. 2006; Northoff and Bermpohl 2004). To date, non-human primates show no sign of this kind of sense of the subjective self (Iriki 2006).

A further example of 'seeing oneself from the outside' is Pickering's (2013) ambush hunting hypothesis. The strategy requires stealth, rudimentary observational skills to find a game trail or waterhole regularly used by intended prey animals, and intentionally setting oneself up in concealment of such prey. Thus to be successful, ambush hunters need to be aware of how they will appear to the prey, which requires an objective body representation. It is currently held that between about 1 million years and 300 thousand years, hominin meat-getting strategies developed 
from advanced scavenging strategies into hunting with rudimentary spears, exploiting ambush situations that placed prey animals at a disadvantage. For example, at Olorgesailie, Kenya, spanning $\sim 1.2$ to 0.5 million years (Kübler et al. 2015), and Elandsfontein, South Africa, at 600 thousand years ago (Bunn 2019), there is evidence that hominins were capable ambush hunters who understood animal use of the landscape, indicating the evolution of grade 5 causal cognition in African populations before their split with the Neanderthals.

The self-objectified perspective has far-reaching consequences. Donald (1991) formulates a 'mimesis hypothesis', which states that a specific form of cognition (and a corresponding culture) mediated between that of the ancestor we have in common with the apes and that of modern humans. In brief, Donald proposes that while ape culture is based on associational learning, early Homo evolved a new form of cognition. The basis for this was that the body could be used 'volitionally' to do what somebody else is doing (imitation), to represent external events for the purpose of communication (mime, gesture), and to rehearse a given skill by matching performance to a goal. We submit that this volitional aspect of an action is made possible by self-objectification, which results in the body of the self becoming part of the detached representations of the inner space. In other words, the body is itself perceived as a tool.

Donald (2012) expands the mimesis hypothesis and emphasizes that a key feature of the human memory system is our ability to voluntarily retrieve a particular memory. He notes: "Nonhuman animals can learn skills with appropriate conditioning, but their performance can be retrieved only by external cues that elicit conditioned responses. Voluntary recall, as in self-triggered conscious retrieval, the kind of recall needed to practice a skill, is absent" (Donald 2012:275). He calls the ability to initiate the internal cuing process that triggers a memory of a previous performance 'autocuing'. Again, autocuing depends on having access to a detached representation of one's actions or seeing one's actions from the outside, since the actions one is performing now must be compared to the actions performed earlier. Autocuing can thus be seen as a direct consequence of self-objectification.

Tool cultures that evolved in the Homo lineage presume a well-established ability to rehearse. For example, Gärdenfors and Högberg (2017) argue that learning to knap Oldowan tools already requires extensive practice. Even though rehearsal is best seen as a form of self-imitation-I remember how I performed a sequence of actions last time and what the outcome was, now I can try to improve on previous performance - it is cognitively more complex than imitation. Imitation only presumes copying of the action of others, while rehearsal depends on autocuing memories, and thereby on a detached representation of one's own actions (Donald 2012).

Traces of self-objectification can be found in apes. Apes, but not monkeys, can recognize themselves in mirrors (e.g. Gallup 1977). Chimpanzees can also use mirrors to guide their hands to grab food that is hidden from direct view (Menzel et al. 1985). By sticking its arm through a hole while looking at the mirror reflection, the chimpanzee could navigate its hand to retrieve the treat, but rhesus macaques failed the same task repeatedly. Such mirror use indicates that apes have self-awareness in the form of body-awareness. However, it does not show that they have a detached representation of their own actions. In fact, 
there is no evidence of apes voluntarily rehearsing a skill. Hihara et al. (2003) have, however, shown that when tool-use training was combined with vocal production learning, monkeys could, with some effort, learn to volitionally give reference calls.

\section{Tool use extends causal thinking using detached representations}

When one uses a hammerstone to crack a nut, the result is something that is immediately edible. When one uses a hammerstone to shape another stone tool, the result is something usable. Cracking the nut opens up for consumption, but a tool opens up for new actions that are detached in time and space (Haidle et al. 2015; Lombard et al. 2019). Among non-human animals, one finds examples of tool manufacture and tool use that seem to be more or less innate (Shumaker et al. 2011).

In contrast, the tool manufacture of hominins was flexible and typically learned by practice. They could not have been made without thinking about the possible actions and their causal consequences. In this way, tool making supports the development of causal thinking. Vice versa, thinking about actions that may lead to a goal opens up for thinking about the potential of different kinds of tools. For example, humans today use the percussive force of a hammer to drive a nail into a wall, but use a sharp-edged axe to chop wood. Although the hammering and chopping actions are similar, the tools and outcomes are different. Also, humans may use the same hammer for a range of purposes such as cracking nuts, driving home nails, flattening a piece of metal or chiseling. We can do this because we understand the force-potential of hammer use abstractly, which enables us to imagine how to apply the technology across a range of problems. In contrast, the hammer-assisted nut cracking of chimpanzees does not seem to lead to thoughts about other uses for the technology at their current stage of cognitive evolution (also see Boesch et al. 2017). The fact that some chimpanzee tribes poke and fish for small prey, termites or honey with sticks, however, indicate their potential for a basic understanding of extended force application.

Building on arguments from embodied cognition, Malafouris (2013) suggests that there is an integration of the extension of the human body through technology and the extension of our cognitive abilities. Bruner and Lozano (2014) further argue that the development of the human mind cannot be decoupled from the use of technology, whereby the body functioning as motor and perceptual interface between internal and external worlds shapes our reasoning. Engagement with technology or material culture is one of the strongest representations of such interfacing, because working with objects provokes certain forms of cognition, intensifies observational and computational capacities, and stores information outside the brain. They therefore suggest that "[w]ithout tools, not only couldn't our mind do what it does, but also it wouldn't even be the way it is" (Bruner and Lozano 2014:273). Our account of how tools extend the peripersonal space forms the basis for an explanation of how this integrality can arise. 


\section{Hominin technology and higher grades of causal thinking}

\section{Action at a distance}

Even further extensions of your peripersonal space are achieved when the tool leaves the direct control of your body and exerts its force at a distance-entering extrapersonal space. In this section we argue that this extension has led to grade 6 causal reasoning about forces.

Throwing an object, which is very common among primates, may be the first method of force transmission at a distance (see Calvin 1993 for a speculative account). When chimpanzees hunt bush babies with sharpened sticks, they thrust using direct force (Preutz and Bertolani 2007). They do not hunt by throwing. Although they have been observed to occasionally throw objects to intimidate, they generally lack the accuracy, force and speed that make human throwing so effective and dangerous (e.g. Roach et al. 2013).

Hopkins et al. (2012) studied individual variation in throwing among a group of 91 chimpanzees. Only 39 of them threw consistently, and these chimpanzees showed greater connectivity between white matter and grey matter in the part of the brain associated the motor-hand area (precentral gyrus and inferior frontal gyrus). Cantalupo and Hopkins (2009) also found that chimpanzees that had learned to throw had significantly larger cerebella than those that had not, and the differences were mainly in the hemisphere opposite to their preferred throwing hand. Interestingly, those that had learned to throw also showed significantly better communicative capacities than the non-throwers.

Hopkins et al. (2012:39) note that the function of the chimpanzees' throwing action is to influence the behaviors of other social beings, including human observers. Furthermore, Leavens et al. (2005) argue that ape gestural communication of captive individuals mainly covers cases where apes point to otherwise unattainable foods in the presence of humans. In this way, they manipulate a human to get them food, using the human as a tool. This means that both throwing and gesturing are 'social tools' as presented by Jacobs and Osvath (2016).

It is also reasonable to accept that, similar to current-day chimpanzees or capuchin monkeys, our early ancestors threw objects to intimidate predators, scavengers, and perhaps other hominins (e.g. Hopkins et al. 2012). As Young (2003:165) explains: "The best throwers and clubbers in a community would rise in the male dominance hierarchy and thereby obtain more breeding opportunities. ... Defense against predators would be enhanced, and opportunities for scavenging would increase when predators could be driven from carcasses". This is an example of the extension or of body and mind through using technology.

During the evolution of hominins, the intimidating function of throwing has increasingly been replaced by a physical function. In hunting or in combat, the aim is to physically hurt or kill the individual thrown at. Therefore, the thrower must find a way to reason about the physical effects, over and above the social ones of a throw. We submit that the result of this process was a detached understanding of force transmitted by the thrown object. 
Not only the shape of the hand, but also the shoulder and humerus changed during the evolution of the hominins in such a way that made throwing more effective in terms of strength and aiming accuracy (Roach et al. 2013, Roach and Lieberman 2014). The anatomical adaptations that enable elastic energy storage and release at the shoulder first appear in their 'modern' configuration in H. erectus about 2 million years ago (Roach et al. 2013). The gluteus maximus is also much expanded in the hominins relative to that of the chimpanzees. This expansion cannot only be explained by the role of the muscle in running, but the big muscle also adds considerable force to a throw (e.g. Stern 1972). Throwing stones or spears accurately and forcefully with the purpose to hurt or kill represents the transmission of arm force away from the direct perception of the thrower. A thrower that does not identify the cause with their bodily action, but mentally represents the force or energy of the spear will be better at understanding the mechanism and results of throwing and thereby better at throwing. Furthermore, with such an understanding it will be possible to generalize to new situations involving force transmission.

Early evidence for such a transfer of knowledge comes from a classical experiment by Judd (1908) in which fifth- and sixthgrade students were instructed to throw darts at a target submerged 12 inches under water. Some of the students were given an explanation of the principle of optical refraction that made the underwater target appear dislocated, and some others were not. On the first task, there was no difference in the success of hitting the target. However, when the depth of the target was changed from 12 to 4 inches, the group that had received the explanation about refraction were far superior to the group that had not. The experiment has been repeated in many variations, beginning with Hendrickson and Schroeder (1941).

We submit that the transition from actions to forces as causes is a critical step in the evolution of causal reasoning associated with grade 6 , inanimate causal understanding. The effect of the stone or spear on prey is also detached in space from the thrower. Furthermore, the mapping between cause and effect must be inferred from the consequences of the throw, for example, from the behavior of an animal that is hit.

Support for high-level cognition associated with well-developed throwing capacity comes from a comparative throwing study using data from humans and capuchin monkeys, showing that the high-performance throwing of humans requires enhanced levels of spatial targeting, precision timing, and multi-joint motor control (Westergaard et al. 2000; Watson 2001).

The individual differences found in extant chimpanzees indicate that the common ancestor to humans and chimpanzees had a potential to develop the throwing capacity. Based on their neurological work with chimpanzees, Hopkins et al. (2012) further proposed that subsequent to the pan-hominin split, there was a strong selection amongst the hominins to increase motor skills associated with throwing that could have initiated hemispheric specialization in the hominin brain.

A neuro-archaeological study also found that elements of the central executive, such as inhibition and focusing on external and internal attention, are used during spear throwing (Williams et al. 2014), suggesting that spear-hunting Neanderthals and $H$. heidelbergensis possessed aspects of such cognitive functioning. Above we suggested that inhibition, or being able to suppress the sensations one has for the 
moment, is necessary for detached representation associated with the causal understanding of forces through time and space. Notions of executive cognitive functioning are therefore intimately linked with high-level causal reasoning. Although it has been suggested that the Neanderthal-associated Schöningen spears were used for throwing (Thieme 1997), effective throwing for hunting purposes is probably better developed in $H$. sapiens compared to more archaic populations. For example, Coolidge et al. (2015:195) suggest that Neanderthals could have been limited in their spear-throwing abilities and thus probably had less well developed "hand/movement control, vision, and spatial awareness and judgment" compared to $H$. sapiens. This inference is palaeo-neurologically supported based on between-species variation in parietal surface morphology, with that of Neanderthals possibly being less extensive than that of $H$. sapiens, implying differences in their visuospatial integration (Bruner and Lozano 2014).

Fully-developed visuospatial integration is key to successful hand-eye coordination, spatial awareness and judgment at a distance, and probably evolved incrementally throughout our genus. Bruner et al. (2017) found that key areas in visuospatial integration such as the precuneus, expanded fully only in $H$. sapiens during the last 150.000 years, and that this expansion may be linked to the cognitive specializations observed in recent humans. We suggest that it is also important for the ability to mentally simulate the causal operation of technologies through time and space in abstract terms and thereby being able to reason about forces acting at a distance.

Grade 6 or inanimate causal cognition also explains the ability to infer the forces of twine or a sticky substance such as tree gum as binding agents for the construction of composite tools such as stone-tipped spears (Gärdenfors and Lombard 2018). Such weapons may have been in occasional use since 500.000 years ago (Wilkins et al. 2012; but see Wadley 2013; Wurz 2013; Rots and Plisson 2014 for issues with context and method), and in wide-spread use since about 300.000 years ago with the proliferation of Levallois stone points and evidence for hafting throughout the Old World (Mazza et al. 2006; Tryon et al. 2005; Barham 2013; Sahle et al. 2013; Niekus et al. 2019).

\section{Using stored forces}

A further type of grade 6 causal thinking involves tools where energy is stored and released when needed. In their review of animal tool use, Shumaker et al. (2011:224) note: "Only humans harness other forms of mechanical energy ... to power their tools". Technologies involving bow-and-arrow sets or snares involve causal reasoning concerning indirect transmission of force via stored energy. This is in contrast with using basic leverage for spear throwing and increased leverage obtained with atlatls/spear-throwers (e.g. Carignani 2016). The mechanical principles of bow hunting require both the understanding of directional force transmission through launching an arrow by drawing the arm backward (in the opposite direction of intended flight, a counter-intuitive action that requires mental inhibition), and the understanding that the stored energy in a bent branch can be used to propel the projectile forward (Lombard and Phillipson 2010). In Gärdenfors and Lombard (2018), we 
have previously argued that bow hunting is a clear example of basic grade 7 causal understanding, indicating minds that are able to apply abstract engineering concepts across at least two different knowledge domains to form a single 'machine'.

The mental simulations involved in spear throwing and to an even higher extent in arrow shooting (Lombard 2019), are related to Donald's (2012) concept of autocuing, implicating the recollection of events and event clusters at will. A clear consciousness of the self in a past memory, and the detached manipulation of such memories could be unique to H. sapiens (Coolidge et al. 2016). Such a memory system would be critical for the development of enhanced self-objectification as described by Iriki (2006), and a cognitive system able to consciously activate, deactivate, and reactivate abstracted knowledge in mental simulations that are detached in space and time.

The neuro-archaeological experiment that compared spear throwing with arrow shooting, also found that shooting arrows showed statistically significant higher levels of neural activity compared with the spear-throwing task (Williams et al. 2014). The arrow-shooting task required a number of action sequences to be remembered, and higher levels of sustained attention and mental rehearsal, enabling the synchronization of abstracted ideas with motor planning and task execution (Lombard 2019; May-Benson and Cermak 2007).

The expansion of the precuneus was possibly important for this type of cognition as its activation is also observed in modern archers when they strain to integrate the relevant sensory and cognitive information whilst planning a successful shot (Chang et al. 2011). Thus, techno-behaviors such as bow hunting (amongst others) may well have contributed to exert selective pressure (on both minds and brains) that increased $H$. sapiens' capacity for integrating our abstracted inner space with the visuospatial challenges and realities of our increasingly complex outer worlds (Lombard 2019).

\section{Forces acting over time}

An even more complex form of causal reasoning about a force operating over an extended period, sometimes across a long distance, and often out of sight, is the use of poisoned arrows where animals are wounded and then tracked for several hours or even days before killed and harvested (Bradfield et al. 2015).

Poison is not a physical force-it functions chemically. In Gärdenfors and Lombard (2018), we have argued that its use adds yet another domain-specific causal node set to that of bow hunting. When preparing and using a poisoned arrow, the hunter must rely on more advanced forms of abstract causal reasoning and planning than thus far discussed. Whereas Boyd (2017), and Henrich (2017), for example, argue that ancient synthetic substances merely represent customary recipes, followed by unreflective tradition, Wadley's (2010) experimental work on adhesive production, and recent ethnographic observations about poison production amongst San hunters of Namibia (Wadley et al. 2015; Chaboo et al. 2016), reveal a different perspective (also see Osiuraka and Reynaud in press). Whilst cognizant of tradition, symbolism and variation through time, these studies demonstrate that such techno-behaviours are far from 
being mechanistic, thoughtless processes that can be explained through, for example, expert cognition (Wynn et al. 2017). Also Osiurak et al. (2020, pp. 3-4) emphasize that humans are not just manipulators, but "physical problem solvers or makers" and that "even if tool-use activites appear to us to be routines, most of them are based on the expertise we have in reasoning about out physical world".

Even though aspects of poison recipes form part of a group's traditions, with a long history of trial and error, they are backed by a wealth of botanical knowledge and adapted to different ecological niches. The collecting of the all different ingredients happens over an extended period, and the manufacturing of the compounds require carefully monitored heat treatment. Such treatment represents a range of different techniques and requires high levels of attention to monitor time exposed to heat and changes to the compounds. During each production session, continuous adjustments are made to the amounts of each ingredient added, so that ultimately the right consistency is achieved, depending on an array of climatic, hunting and other conditions. The observed skill to produce toxins for poisoned arrows effectively therefore implies long attention spans, response inhibition, the capacity for novel, sustained multilevel operations, the use of abstract thought, and the ability to plan the assembly of ingredients as well as complex action sequences (Bradfield et al. 2015). What is more, although a certain ingredient may be a constant amongst certain groups, the recipes are not always the same, and different hunters prepare the similar sets of ingredients differently (Wadley et al. 2015; Chaboo et al. 2016). Thus, notwithstanding elements of cultural conditioning, the manufacturing of poisonous compounds for bow hunting necessitates deliberate causal reflection.

It is therefore our position that techno-behaviors such as hunting with poisoned arrows represent a deep network of abstracted causal reasoning similar in complexity to the modern human mind of today and such behaviors are therefore examples of grade 7 causal reasoning.

Such techno-behaviors require reasoning and problem-solving abilities that represent and integrate complex relationships (Kroger et al. 2002). They also require the ability to imagine a range of potential outcomes, to understand the consequences of those outcomes, and to grasp that the outcomes may have been different if any of the preceding circumstances varied (i.e., counterfactual thinking), for which the neural hardware only reaches its final development in humans after adolescence (e.g. Baird and Fugelsang 2004). Interestingly, neuro-maturational changes associated with visuospatial causal reasoning also shift from a widespread frontal pattern in childhood to predominant, integrated frontoparietal activation in late adolescence (e.g. Eslinger et al. 2009). In combination with Bruner's evidence for the relative late enlargement of the complete parietal surface and the precuneus in hominin evolution (Bruner 2010; Bruner et al. 2017), these observations may explain why techno-behaviors such as bow hunting are unique to $H$. sapiens. 


\section{Conclusion}

Here we explored how technologies that expand our technical thinking beyond our immediate perceptual input may have impacted on human cognitive evolution. We have demonstrated that the human understanding of abstract forces, and imagining how they can be used in novel ways, is unique compared to other primates. As an analytic tool, we have used the seven grades of causal cognition from Lombard and Gärdenfors (2017). In this article we have focused on explaining how grade 6 causal reasoning about forced has evolved.

The use of complex technologies during the Stone Age, such as hunting with bows and poisoned arrows, led us to infer high grades of understanding abstract causality. This development came about as a result of the coevolution of long-term technical engagement (e.g. Barham 2013; Shea 2017), and incremental but not necessarily unilinear improvements in causal understanding throughout the hominin evolutionary history (e.g. Lombard and Gärdenfors 2017; Lombard et al. 2019).

The main mechanism for this expansion builds on results showing that tool use expands action field and action control. As a result, the agent can form a detached representation of itself, which in turn enables what Donald (2012) calls autocuing and thereby a capacity to rehearse. The agent learns to not only perceive actions, but also to represent the forces involved. Forces then function as central variables in the mental simulations of causal relations that also are extended to non-animate events (see also Martin 2014). We can summarize this position by saying that with the aid of our causal reasoning we can use our tools to solve new physical problems (see also Osiurak et al. 2020). Although much work remains to fill in this proposed evolutionary progression of causal reasoning, it is encouraging that palaeo-neurological work as well as independent neurological investigations support this account.

Acknowledgements Open access funding provided by Lund University. The authors gratefully acknowledge support from Stellenbosch Institute for Advanced Study (STIAS). PG also thanks the Swedish Research Council for support to the Linnaeus environment Thinking in Time: Cognition, Communication and Learning.

Open Access This article is licensed under a Creative Commons Attribution 4.0 International License, which permits use, sharing, adaptation, distribution and reproduction in any medium or format, as long as you give appropriate credit to the original author(s) and the source, provide a link to the Creative Commons licence, and indicate if changes were made. The images or other third party material in this article are included in the article's Creative Commons licence, unless indicated otherwise in a credit line to the material. If material is not included in the article's Creative Commons licence and your intended use is not permitted by statutory regulation or exceeds the permitted use, you will need to obtain permission directly from the copyright holder. To view a copy of this licence, visit http://creativecommons.org/licen ses/by/4.0/.

\section{References}

Ambrose SH (2001) Paleolithic technology and human evolution. Science 291:1748-1753

Arbib MA, Bonaiuto JB, Jacobs S, Frey SH (2009) Tool use and the distalization of the end-effector. Psychol Res 73:441-462 
Baird AA, Fugelsang JA (2004) The emergence of consequential thought: evidence from neuroscience. Philos Trans R Soc B Biol Sci 359:1797-1804

Barbey AK, Colom R, Solomon J, Krueger F, Forbes C, Grafman J (2012) An integrative architecture for general intelligence and executive function revealed by lesion mapping. Brain 135:1154-1164

Barham L (2013) From hand to handle: the first industrial revolution. Oxford University Press, Oxford

Boesch C, Bombjaková D, Boyette A, Meier A (2017) Technical intelligence and culture: nut cracking in humans and chimpanzees. Am J Phys Anthropol 163:339-355

Boivin N (2008) Material cultures, material minds: the impact of things on human thought, society, and evolution. Cambridge University Press, Cambridge

Boyd R (2017) A different kind of animal: how culture transformed our species. Princeton University Press, Princeton

Bradfield J, Lombard M, Wadley L (2015) Southern African arrow poison recipes, their ingredients and implications for Stone Age archaeology. South Afr Humanit 27:29-64

Bruner E (2010) Morphological differences in the parietal lobes within the human genus: a neurofunctional perspective. Curr Anthropol 51(S1):S77-S88

Bruner E, Lozano M (2014) Extended mind and visuo-spatial integration: three hands for the Neandertal lineage. J Anthropol Sci 92:273-280

Bruner E, Preuss T, Chen X, Rilling K (2017) Evidence for expansion of the precuneus in human evolution. Brain Struct Funct 222:1053-1060

Bunn H (2019) Large ungulate mortality profiles and ambush hunting by Acheulean-age hominins at Elandsfontein, Western Cape Province, South Africa. J Archaeol Sci 107:40-49

Cacchione T, Call J, Zingg R (2009) Gravity and solidity in four great ape species (Gorilla gorilla, Pongo pygmaeus, Pan troglodytes, Pan paniscus): vertical and horizontal variations of the table task. $\mathrm{J}$ Comp Psychol 123:168-180

Call J (2010) Trapping the minds of apes: causal knowledge and inferential reasoning about object-object interactions. In: Lonsdorf EV, Ross S, Matsuzawa T (eds) The mind of the chimpanzee: ecological and experimental perspectives. University of Chicago Press, Chicago, pp 75-86

Calvin W (1993) The unitary hypothesis: a common neural circuitry for novel manipulations, language, plan-ahead, and throwing. In: Gibson K, Ingold T (eds) Tools, language, and cognition in human evolution. Cambridge University Press, Cambridge, pp 230-250

Cantalupo C, Hopkins W (2009) The cerebellum and its contribution to complex tasks in higher primates: a comparative perspective. Cortex 46:821-830

Carignani G (2016) On the origin of technologies: the invention and evolution of the bow-and-arrow. In: Panebianco F, Serrelli E (eds) Understanding cultural traits. Springer, Berlin, pp 315-339

Chaboo CS, Biesele M, Hitchcock RK, Weeks A (2016) Beetle and plant arrow poisons of the Jul'hoan and Haill om San peoples of Namibia (Insecta, Coleoptera, Chrysomelidae; Plantae, Anacardiaceae, Apocynaceae, Burseraceae). ZooKeys 558:9

Chang Y, Lee J-J, Seo J-H, Song H-J, Kim Y-T, Lee H, Kim H, Lee J, Kim W, Woo M, Kim J (2011) Neural correlates of motor imagery for elite archers. NMR Biomed 24:366-372

Cheney D, Seyfarth R (1990) Attending to behaviour versus attending to knowledge: examining monkeys' attribution of mental states. Anim Behav 40:742-753

Civelek Z, Call J, Seed A (2020) Inferring unseen causes: developmental and evolutionary origins. Front Psychol 11:872. https://doi.org/10.3389/fpsyg.2020.00872

Cléry J, Guipponi O, Wardak C, Hamed S (2015) Neuronal bases of peripersonal and extrapersonal spaces, their plasticity and their dynamics: knowns and unknowns. Neuropsychologia 70:313-332

Coolidge FL, ThomasWynn T, Overmann KA, Hicks JM (2015) Cognitive archaeology and the cognitive sciences. In: Bruner E (ed) Human paleoneurology. Springer, Berlin, pp 177-208

Coolidge F, Haidle M, Lombard M, Wynn T (2016) Bridging theory and bow hunting: human cognitive evolution and archaeology. Antiquity 90:219-228

D’Argembeau A, Feyers D, Majerus S, Collette F, Van der Linden M, Maquet P, Salmon E (2008) Selfreflection across time: cortical midline structures differentiate between present and past selves. Soc Cogn Affect Neurosci 3:244-252

Decety J, Grèzes J (2006) The power of simulation: imagining one's own and other's behavior. Brain Res 1079:4-14

Donald M (1991) Origins of the modern mind: three stages in the evolution of culture and cognition. Harvard University Press, Cambridge 
Donald M (2012) Evolutionary origins of autobiographical memory: a retrieval hypothesis. In: Berntsen D, Rubin D (eds) Understanding autobiographical memory: theories and approaches. Cambridge University Press, Cambridge, pp 269-289

Doricchi F, de Schotten M, Tomaiuolo F, Bartolomeo P (2008) White matter (dis) connections and gray matter (dys) functions in visual neglect: gaining insights into the brain networks of spatial awareness. Cortex 44:983-995

Eslinger P, Blair C, Wang J, Lipovsky B, Realmuto J, Baker D, Thorn S, Gamson D, Zimmerman E, Rohrer L, Yang Q (2009) Developmental shifts in fMRI activations during visuospatial relational reasoning. Brain Cogn 69:1-10

Gallup G (1977) Self recognition in primates: a comparative approach to the bidirectional properties of consciousness. Am Psychol 32:329-338

Gärdenfors P (2003) How Homo became Sapiens: on the evolution of thinking. Oxford University Press, Oxford

Gärdenfors P (2007) Evolutionary and developmental aspects of intersubjectivity. In: Liljenström H, Århem P (eds) Consciousness transitions: phylogenetic, ontogenetic and physiological aspects. Elsevier, Amsterdam, pp 281-305

Gärdenfors P, Högberg A (2017) The archaeology of teaching and the evolution of Homo docens. Curr Anthropol 58:188-201

Gärdenfors P, Lombard M (2018) Causal cognition, force dynamics and early hunting technologies. Front Psychol 9:87. https://doi.org/10.3389/fpsyg.2018.00087

Gärdenfors P, Osvath M (2010) Prospection as a cognitive precursor to symbolic communication. In: Larson R, Déprez V, Yamakido H (eds) Evolution of language: biolinguistic approaches. Cambridge University Press, Cambridge, pp 103-114

Gärdenfors P, Warglien M (2012) Using conceptual spaces to model actions and events. J Semant 29:487-519

George N, Göksun T, Hirsh-Pasek K, Golinkoff R (2019) Any way the wind blows: children's inferences about force and motion events. J Exp Child Psychol 177:119-131

Glenberg A (1997) What memory is for. Behav Brain Sci 20:1-19

Göksun T, George N, Hirsh-Pasek K, Golinkoff R (2013) Forces and motion: how young children understand causal events. Child Dev 84:1285-1295

Goldenberg G, Spatt J (2009) The neural basis of tool use. Brain 132:1645-1655

Gopnik A, Meltzoff A, Kuhl P (1999) The scientist in the crib: minds, brains, and how children learn. William Morrow and Co, New York

Gopnik A, Glymour C, Sobel D, Schultz L, Kushnir T, Danks D (2004) A theory of causal learning in children: causal maps and Bayes nets. Psychol Rev 111:3-32

Grush R (1997) The architecture of representation. Philos Psychol 10:5-23

Haidle M, Bolus M, Collard M, Conard N, Garofoli D, Lombard M, Nowell A, Tennie C, Whiten A (2015) The nature of culture: an eight-grade model for the evolution and expansion of cultural capacities in hominins and other animals. J Anthropol Sci 93:43-70

Hampshire A, Thompson R, Duncan J, Owen A (2010) Lateral prefrontal cortex subregions make dissociable contributions during fluid reasoning. Cereb Cortex 21:1-10

Hannah AC, McGrew WC (1987) Chimpanzees using stones to crack open oil palm nuts in Liberia. Primates 28(1):31-46

Hanus D, Call J (2008) Chimpanzees infer the location of a reward on the basis of the effect of its weight. Curr Biol 18:R370-R372

Harmand S, Lewis J, Feibel C, Lepre C, Prat S, Lenoble A, Boës X, Quinn R, Brenet M, Arroyo A, Taylor N, Clément S, Daver G, Brugal J-P, Leakey L, Mortlock R, Wright J, Lokorodi S, Kirwa C, Kent D, Roche H (2015) 3.3-million-year-old stone tools from Lomekwi 3, West Turkana, Kenya. Nature 521:310-315

Hendrickson G, Schroeder WH (1941) Transfer of training in learning to hit a submerged target. J Educ Psychol 32:205-213

Henrich J (2017) The secret of our success: how culture is driving human evolution, domesticating our species, and making us smarter. Princeton University Press, Princeton

Hihara S, Yamada H, Iriki A, Okanoya K (2003) Spontaneous vocal differentiation of coo-calls for tools and food in Japanese monkeys. Neurosci Res 45:383-389

Hopkins W, Russell J, Schaeffer J (2012) The neural and cognitive correlates of aimed throwing in chimpanzees: a magnetic resonance image and behavioural study on a unique form of social tool use. Philos Trans R Soc Lond B Biol Sci 367(1585):37-47 
Iriki A (2006) The neural origins and implications of imitation, mirror neurons and tool use. Curr Opin Neurobiol 16:660-667

Iriki A, Taoka M (2012) Triadic (ecological, neural, cognitive) niche construction: a scenario of human brain evolution extrapolating tool use and language from the control of reaching actions. Philos Trans R Soc Lond B Biol Sci 367(1585):10-23

Jacobs I, Osvath M (2016) Social tool. In: Weekes-Shackelford VA, Shackelford TK (eds) Encyclopedia of evolutionary psychological science. Springer, Berlin. https://doi.org/10.1007/978-3-319-16999 $-6 \_3157-1$

Jelbert S, Miller R, Schiesti M, Boeckle M, Cheke L, Gray R, Taylor A, Clayton N (2019) New Caledonian crows infer the weight of objects from observing their movements in a breeze. Proc R Soc B 286(1894):20182332

Johnson M, Raye C, Mitchell K, Touryan S, Greene E, Nolen-Hoeksema S (2006) Dissociating medial frontal and posterior cingulate activity during self-reflection. Soc Cogn Aff Neurosci 1:56-64

Johnson-Frey S (2003) What's so special about human tool use? Neuron 39:201-204

Johnson-Frey S (2004) The neural bases of complex tool use in humans. Trends Cogn Sci 8:71-78

Judd CH (1908) The relation of special training to general intelligence. Educ Rev 36:28-42

Köhler W (1917) The mentality of apes. [Trad. Ella Winter]. Penguin Books, Michigan

Krawczyk D (2012) The cognition and neuroscience of relational reasoning. Brain Res 1428:13-23

Kroger JK, Sabb FW, Fales CL, Bookheimer SY, Cohen MS, Holyoak KJ (2002) Recruitment of anterior dorsolateral prefrontal cortex in human reasoning: a parametric study of relational complexity. Cerebral Cortex 12:477-485

Kübler S, Owenga P, Reynolds S, Rucina S, King G (2015) Animal movements in the Kenya Rift and evidence for the earliest ambush hunting by hominins. Sci Rep 5:14011

Leavens D, Hopkins W, Bard K (2005) Understanding the point of chimpanzee pointing: Epigenesis and ecological validity. Curr Dir Psychol Sci 14:185-189

Leslie A (1987) Pretense and representation: the origins of 'theory of mind'. Psychol Rev 94:412-426

Leslie AM (1995) A theory of agency. In: Sperber D, Premack D, Premack AJ (eds) Causal cognition: a multidisciplinary debate. Oxford University Press, New York, pp 121-141

Leslie A, Keeble S (1987) Do six-month-old infants perceive causality? Cognition 25:265-288

Lombard M (2016) Mountaineering or ratcheting? Stone Age hunting weapons as proxy for the evolution of human technological, behavioral and cognitive flexibility. In: Haidle MN, Conard NJ, Bolus M (eds) The nature of culture. Springer, Berlin, pp 135-146

Lombard M (2019) On the minds of bow hunters. In: Coolidge FL, Overmann KA, Wynn T (eds) Squeezing minds from stones. University of Oxford Press, Oxford, pp 473-496

Lombard M, Gärdenfors P (2017) Tracking the evolution of causal cognition in humans. J Anthropol Sci 95:219-234

Lombard M, Gärdenfors P (submitted) Causal cognition and theory of mind in evolutionary cognitive archaeology

Lombard M, Phillipson L (2010) Indications of bow and stone-tipped arrow use 64000 years ago in KwaZulu-Natal, South Africa. Antiquity 84:635-648

Lombard M, Högberg A, Haidle MN (2019) Cognition: from Capuchin rock pounding to Lomekwian Flake production. Camb Archaeol J 29:201-231. https://doi.org/10.1017/S0959774318000550

Malafouris L (2013) How things shape the mind. MIT Press, Cambridge

Maravita A, Iriki A (2004) Tools for the body (schema). Trends Cogn Sci 8:79-86

Martin S-F (2014) The origins of causal cognition in early hominins. Biol Philos 30:247-266

Martin-Ordas G, Call J, Colmenares F (2008) Tubes, tables and traps: great apes solve two functionally equivalent trap tasks but show no evidence of transfer across tasks. Anim Cogn 11:423-430

Matsuno T, Tomonagab M (2017) Causal capture effects in chimpanzees (Pan troglodytes). Cognition 258:153-164

May-Benson T, Cermak S (2007) Development of an assessment for ideational praxis. Am J Occup Ther 61:148-153

Mazza P, Martini F, Sala B, Magi M, Colombini M, Giachi G, Landucci F, Lemorini C, Modugno F, Ribechini E (2006) A new Palaeolithic discovery: Tar-hafted stone tools in a European Mid-Pleistocene bonebearing bed. J Archaeol Sci 33:1310-1318

Menzel EW Jr, Sue Savage-Rumbaugh E, Lawson J (1985) Chimpanzee (Pan troglodytes) spatial problem solving with the use of mirrors and televised equivalents of mirrors. J Comp Psychol 99:211

Merleau-Ponty M (1962) Phenomenology of perception [Phénoménologie de la Perception]. Routledge and Kegan Paul, London 
Michotte A (1963) The perception of causality. Methuen, New York

Morgan BJ, Abwe EE (2006) Chimpanzees use stone hammers in Cameroon. Curr Biol 16(16):R632-R633

Mulcahy N, Call J (2006) How great apes perform on a modified trap-tube task. Anim Cogn 9:193-199

Niekus M, Kozowyk P, Langejans G, Ngan-Tillard D, van Keulen H, van der Plicht J, Cohen K, van Wingerden W, van Os B, Smit B, Amkreutz L, Johansen L, Verbaas A, Dusseldorp G (2019) Middle Paleolithic complex technology and a Neandertal tar-backed tool from the Dutch North Sea. Proc Natl Acad Sci 116:22081-22087

Northoff G, Bermpohl F (2004) Cortical midline structures and the self. Trends Cogn Sci 8:102-107

Osiurak F, E Reynaud (in press) The elephant in the room: what matters cognitively in cumulative technological culture. Behav Brain Sci. 10.1017/s0140525x19003236

Osiurak F, Lesourd M, Navarro J, Reynaud E (2020) Technition: when tools come out of the closet. Perspect Psuchol Sci 15:880-897

Penn D, Povinelli D (2007) Causal cognition in human and nonhuman animals: a comparative, critical review. Annu Rev Psychol 58:97-118

Pickering T (2013) Rough and tumble: aggression, hunting, and human evolution. University of California Press, Berkeley

Povinelli D (2000) Folk physics for apes: the chimpanzee's theory of how the world works. Oxford University Press, Oxford

Povinelli DJ, Penn DC (2011) Through a floppy tool darkly Toward a conceptual overthrow of animal alchemy. In: McCormack TT, Hoerl C, Butterfill S (eds) Tool use and causal cognition. Oxford University Press, Oxford, pp 69-88

Preutz J, Bertolani P (2007) Savanna chimpanzees, Pan troglodytes verus, hunt with tools. Curr Biol 17:412-417

Rizzolatti G, Craighero L (2004) The mirror-neuron system. Annu Rev Neurosci 27:169-192

Roach N, Lieberman D (2014) Upper body contributions to power generation during rapid, overhand throwing in humans. J Exp Biol 217:2139-2149

Roach N, Venkadesan M, Rainbow M, Lieberman D (2013) Elastic energy storage in the shoulder and the evolution of high-speed throwing in Homo. Nature 498:483-487

Rots V, Plisson H (2014) Projectiles and the abuse of the use-wear method in a search for impact. J Archaeol Sci 48:154-165

Sahle Y, Hutchings WK, Braun DR, Sealy DR, Morgan LE, A Negash, Atnafu B (2013) Earliest stonetipped projectiles from the Ethiopian Rift date to $>279,000$ years ago. PLoS ONE 8(11):e78092

Sanz C, Morgan D, Gulick S (2004) New insights into chimpanzees, tools, and termites from the Congo Basin. Am Nat 164:567-581

Seed A, Call J, Emery N, Clayton N (2009) Chimpanzees solve the trap problem when the confound of tool-use is removed. J Exp Psychol Anim Behav Process 35:23-34

Seed AM, Hanus D, Call J (2011) Causal knowledge in corvids, primates and children: more than meets the eye? In: McCormack TT, Hoerl C, Butterfill S (eds) Tool use and causal cognition. Oxford University Press, Oxford, pp 89-110

Shea J (2017) Occasional, obligatory, and habitual stone tool use in hominin evolution. Evol Anthropol 26:200-217

Shumaker R, Walkup K, Beck B (2011) Animal tool behavior: the use and manufacture of tools by animals. JHU Press, Baltimore

Spreng RN, Grady CL (2010) Patterns of brain activity supporting autobiographical memory, prospection, and theory of mind, and their relationship to the default mode network. J Cogn Neurosci 22:1112-1123

Stern JT Jr (1972) Anatomical and functional specializations of the human gluteus maximus. Am J Phys Anthropol 36:315-339

Taylor A, Hunt G, Medina F, Gray R (2009) Do New Caledonian crows solve physical problems through causal reasoning? Proc R Soc B 276:247-254

Tenenbaum JB, Niyogi S (2003) Learning causal laws. In: Proceedings of the twenty-fifth annual conference of the cognitive science society, vol 25, pp 1152-1157

Thieme H (1997) Lower Palaeolithic hunting spears from Germany. Nature 385:807-810

Tomasello M, Carpenter M, Call J, Behne T, Moll H (2005) Understanding and sharing intentions: the origins of cultural cognition. Behav Brain Sci 28:675-691

Tomonaga M, Imura T, Mizuno Y, Tanaka M (2007) Gravity bias in young and adult chimpanzees (Pan troglodytes): tests with a modified opaque-tubes task. Dev Sci 10:411-421 
Tryon CA, McBrearty S, Pierre-Jean T (2005) Levallois lithic technology from the Kapthurin formation, Kenya: Acheulian origin and Middle Stone Age diversity. Afr Archaeol Rev 22:199-229

Umiltà MA, Escola L, Intskirveli I, Grammont F, Rochat M, Caruana F, Jezzini V, Rizzolatti G (2008) When pliers become fingers in the monkey motor system. Proc Natl Acad Sci USA 105(6):2209-2213

Villmoare B, Kimbel W, Seyoum C, Campisano C, DiMaggio E, Rowan J, Braun D, Ramón Arrowsmith J, Reed K (2015) Early Homo at 2.8 Ma from Ledi-Geraru, Afar, Ethiopia. Science 347:1352-1355

Völter C, Call J (2014) Great apes (Pan paniscus, Pan troglodytes, Gorilla gorilla, Pongo abelii) follow visual trails to locate hidden food. J Comp Psychol 128:199-208

Wadley L (2010) Were snares and traps used in the Middle Stone Age and does it matter? A review and a case study from Sibudu, South Africa. J Hum Evol 58:179-192

Wadley L (2013) Recognizing complex cognition through innovative technology in Stone Age and Palaeolithic sites. Camb Archaeol J 23:163-183

Wadley L, Trower G, Backwell L, d'Errico F (2015) Traditional glue, adhesive and poison used for composite weapons by Ju/'hoan San in Nyae Nyae, Namibia. Implications for the evolution of hunting equipment in prehistory. PLoS ONE 10:10

Watson N (2001) Sex differences in throwing: monkeys having a fling. Trends Cogn Sci 5:98-99

Westergaard G, Liv C, Haynie M, Suomi S (2000) A comparative study of aimed throwing by monkeys and humans. Neuropsychologia 38:1511-1517

Whitesides GH (1985) Nut cracking by wild chimpanzees in Sierra Leone, West Africa. Primates 26(1):91-94

Wilkins J, Schoville B, Brown K, Chazan M (2012) Evidence for early hafted hunting technology. Science 338(6109):942-946

Williams V, Burke A, Lombard M (2014) Throwing spears and shooting arrows: preliminary results of a pilot neuroarchaeological study. S Afr Archaeol Bull 69:199-207

Wolff P (2007) Representing causation. J Exp Psychol Gen 136:82-111

Wolff P (2008) Dynamics and the perception of causal events. In: Thomas S, Zacks J (eds) Understanding events: how humans see, represent, and act on events. Oxford University Press, Oxford, pp 555-587

Wolff P, Shepard J (2013) Causation, touch, and the perception of force. In: Ross BH (ed) The psychology of learning and motivation, vol 58. Academic Press, New York, pp 167-202

Wolff P, Thorstad R (2017) Force dynamics. In: Waldmann MR (ed) The Oxford handbook of causal reasoning. Oxford University Press, New York, pp 147-167

Woodward J (2011) A philosopher looks at tool use and causal understanding. In: McCormack TT, Hoerl C, Butterfill S (eds) Tool use and causal cognition. Oxford University Press, Oxford, pp 18-50

Wurz S (2013) Technological trends in the Middle Stone Age of South Africa between MIS 7 and MIS 3. Curr Anthropol 54(S8):S305-S319

Wynn T, Haidle MN, Lombard M, Coolidge F (2017) The expert cognition model in human evolutionary studies. In: Wynn TG, Coolidge FL (eds) Cognitive models in paleolithic archaeology. Oxford University Press, New York, pp 21-44

Young R (2003) Evolution of the human hand: the role of throwing and clubbing. J Anat 202:165-174

Publisher's Note Springer Nature remains neutral with regard to jurisdictional claims in published maps and institutional affiliations. 